\title{
Improved Undecidability Results for Reachability Games on Recursive Timed Automata
}

\author{
Shankara Narayanan Krishna Lakshmi Manasa Ashutosh Trivedi \\ Indian Institute of Technology Bombay, Mumbai, INDIA \\ \{krishnas, manasa, trivedi\}@cse.iitb.ac.in
}

\begin{abstract}
We study reachability games on recursive timed automata (RTA) that generalize Alur-Dill timed automata with recursive procedure invocation mechanism similar to recursive state machines. It is known that deciding the winner in reachability games on RTA is undecidable for automata with two or more clocks, while the problem is decidable for automata with only one clock. Ouaknine and Worrell recently proposed a time-bounded theory of real-time verification by claiming that restriction to bounded-time recovers decidability for several key decision problem related to real-time verification. We revisited games on recursive timed automata with time-bounded restriction in the hope of recovering decidability. However, we found that the problem still remains undecidable for recursive timed automata with three or more clocks. Using similar proof techniques we characterize a decidability frontier for a generalization of RTA to recursive stopwatch automata.
\end{abstract}

\section{Introduction}

Timed automata, introduced by Alur and Dill [4], extend finite state machines with a finite set of continuous variables called clocks that grow with uniform rate in each state. Since the syntax of timed automata allows guarding the transitions and states with simple constraints on clocks and resetting clocks during a transition, timed automata can express complex timing based properties of real-time systems. The seminal paper of timed automata [3] showed the decidability of the fundamental reachability problem for the timed automata, that paved the way for the success of timed automata as the specification and verification formalism for real-time systems.

Recursive timed automata (RTAs) [9] extend timed automata with recursion to model real-time software systems. Formally, an RTA is a finite collection of components where each component is a timed automaton that in addition to making transitions between various states, can have transitions to "boxes" that are mapped to other components modeling a potentially recursive call to a component. During such invocation a limited information can be passed through clock values from the "caller" component to the "called" component via two different mechanism: a) pass-by-value, where upon returning from the called component a clock assumes the value prior to the invocation, and b) pass-by-reference, where upon return a clock reflects any changes to the value inside the invoked procedure. The reachability problem for RTA is known [9] to be undecidable for RTA with three or more clocks.

In this paper we study reachability games on recursive timed automata that are played between two players-called Player 1 and Player 2-who take turns to move a token along the infinite graph of configurations (context, states, and clock valuations) of recursive timed automata. In a reachability game the goal of Player 1 is to reach a desirable set of target states, while the goal of Player 2 is to avoid it. The reachability game problem is to decide the winner in a reachability game.

Example 1 The visual presentation of a reachability game on recursive timed automaton with two components $M_{1}$ and $M_{2}$, and one clock variable $x$ is shown in Figure 1 (inspired by example in [9]) where

Adriano Peron and Carla Piazza (Eds.):

Proceedings of the Fifth International Symposium on

Games, Automata, Logics and Formal Verification (GandALF 2014)

EPTCS 161, 2014, pp. 245-259 doi 10.4204/EPTCS.161.21 

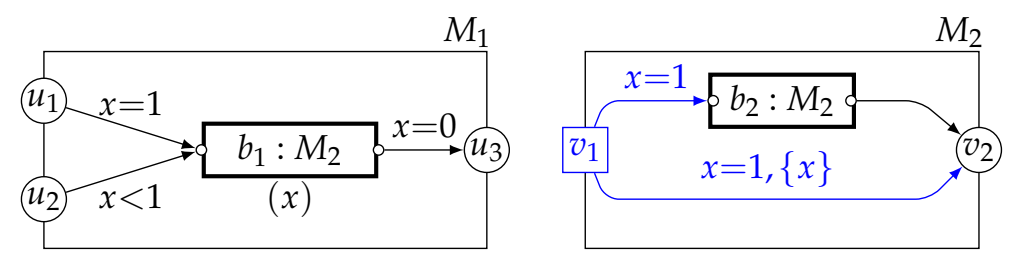

Figure 1: Reachability game on a recursive timed automata with one clock and two components

component $M_{1}$ calls component $M_{2}$ via box $b_{1}$ and component $M_{2}$ recursively calls itself via box $b_{2}$. Components are shown as thinly framed rectangles with their names written next to upper right corner. Various control states, or "nodes", of the components are shown as circles or blue squares with their labels written inside them, e.g. see node $u_{1}$. The circles are Player 1 states (see $\left.u_{1}\right)$ while blue squares $\left(v_{1}\right)$ are Player 2 states. Entry nodes of a component appear on the left of the component (see $\left.u_{1}\right)$, while exit nodes appear on the right (see $\left.u_{3}\right)$. Boxes are shown as thickly framed rectangles inside components labelled $b: M$, where $b$ is the label of the box, $M$ is the component it is mapped to. The tuple of clocks passed to $M$ by value, if any, are shown below the box, and the rest of the variables are passed by reference. For example, in the figure clock $x$ is passed during the invocation of component $M_{2}$ via box $b_{1}$, while no clock is passed by value to component $M_{2}$ via box $b_{1}$. Each transition is labelled with a guard and the set of reset variables, (e.g. transition from node $v_{1}$ to $v_{2}$ can be taken only when variable $x<1$, and after taking this transition, variable $x$ is reset). To minimize clutter we omit empty reset sets.

Trivedi and Wojtczak [9] showed that the reachability game and termination (reachability with empty calling context) game problems are undecidable for RTAs with two or more clocks. Moreover, they considered the so-called glitch-free restriction of RTAs-where at each invocation either all clocks are passed by value or all clocks are passed by reference- and showed that the reachability (and termination) is EXPTIME-complete for RTAs with two or more clocks. In the model of [9] it is compulsory to pass all the clocks at every invocation with either mechanism. Abdulla, Atig, and Stenman [1] studied a related model called timed pushdown automata where they disallowed passing clocks by value. On the other hand, they allowed clocks to be passed either by reference or not passed at all (in that case they are stored in the call context and continue to tick with the uniform rate). It is shown in [1] that the reachability problem for this class remains decidable (EXPTIME-complete). In this article, we restrict ourselves to the recursive timed automata model as introduced in [9].

Ouaknine and Worrell [8] proposed a thesis that restriction to bounded-time recovers decidability for several key decision problem related to real-time verification. In support of this thesis a number of important undecidable problems have been shown to be decidable under bounded-time restriction, for instance language inclusion for timed automata, emptiness problem for alternating timed automata, and emptiness problem for rectangular hybrid automata. The goal of this work was to approach reachability games on recursive timed automata from this viewpoint and to recover the decidability of these games under time-bounded restriction. However, we discovered a rather negative result. In this paper we show that the problem stays undecidable for RTA with just 3 or more clocks.

We also consider the extension of RTAs with stopwatches (clocks that can be paused) to recursive stopwatch automata (RSAs) and show that the time-bounded reachability game problem stays undecidable even for RSAs with 3 or more stopwatches, while we show decidability of glitch-free RSAs with 2 stopwatches. We also show that the reachability problem is undecidable for unrestricted RSA with two or more stopwatches. For the time-bounded reachability case, we show that the problem stays undecidable even for glitch-free variant of RSAs with 4 or more stopwatches. The Table 1 highlights our 


\begin{tabular}{lcccc}
\hline & \multicolumn{2}{c}{ Recursive Timed Automata } & \multicolumn{2}{c}{ Recursive Stopwatch Automata } \\
& TUB & TB & TUB & TB \\
\hline Glitch-free & D & D & U $(\geq 3 \mathrm{sw})$ & $\mathbf{U}(\geq 4 \mathrm{sw})$ \\
& & & $\mathbf{D}(\leq 2 \mathrm{sw})$ & $\mathbf{D}(\leq 2 \mathrm{sw})$ \\
\hline Unrestricted & $\mathrm{U}(\geq 2$ clocks $)$ & $\mathbf{U}(\geq 3$ clocks $)$ & $\mathbf{U}(\geq 2 \mathrm{sw})$ & $\mathbf{U}(\geq 3 \mathrm{sw})$ \\
\hline
\end{tabular}

Table 1: Summary of the contributions of this paper. Results shown in bold are contributions from this paper, while results shown in gray color are from [9]. Here TUB and TB stand for time-unbounded and time-bounded reachability games, and $\mathrm{U}$ stands for undecidable, D for decidable, and sw for stopwatches.

contributions related to reachability games on recursive timed and stopwatch automata. For a survey of models related to RTA and dense-time pushdown automata we refer the reader to [9] and [1]. Due to space limitations, we only sketch the key proofs and details can be found in [6].

\section{Preliminaries}

\subsection{Reachability Games on Labelled Transition Systems.}

A labelled transition system (LTS) is a tuple $\mathcal{L}=(S, A, X)$ where $S$ is the set of states, $A$ is the set of actions, and $X: S \times A \rightarrow S$ is the transition function. We say that an LTS $\mathcal{L}$ is finite (discrete) if both $S$ and $A$ are finite (countable). We write $A(s)$ for the set of actions available at $s \in S$, i.e., $A(s)=\{a: X(s, a) \neq \varnothing\}$. A game arena $G$ is a tuple $\left(\mathcal{L}, S_{1}, S_{2}\right)$, where $\mathcal{L}=(S, A, X)$ is an LTS, $S_{1} \subseteq S$ is the set of states controlled by player Player 1 , and $S_{2} \subseteq S$ is the set of states controlled by Player 2. Moreover, sets $S_{1}$ and $S_{2}$ form a partition of the set $S$. In a reachability game on $G$, rational players-Player 1 and Player 2-take turns to move a token along the states of $\mathcal{L}$. The decision to choose the successor state is made by the player controlling the current state. The objective of Player 1 is to eventually reach certain states, while the objective of Player 2 is to avoid them forever.

We say that $\left(s, a, s^{\prime}\right) \in S \times A \times S$ is a transition of $\mathcal{L}$ if $s^{\prime}=X(s, a)$ and a run of $\mathcal{L}$ is a sequence $\left\langle s_{0}, a_{1}, s_{1}, \ldots\right\rangle \in S \times(A \times S)^{*}$ such that $\left(s_{i}, a_{i+1}, s_{i+1}\right)$ is a transition of $\mathcal{L}$ for all $i \geq 0$. We write Runs $^{\mathcal{L}}$ $\left(F R u n s^{\mathcal{L}}\right)$ for the sets of infinite (finite) runs and $\operatorname{Runs}^{\mathcal{L}}(s)\left(F R u n s^{\mathcal{L}}(s)\right.$ ) for the sets of infinite (finite) runs starting from state $s$. For a set $F \subseteq S$ and a run $r=\left\langle s_{0}, a_{1}, \ldots\right\rangle$ we define $\operatorname{Stop}(F)(r)=$ $\inf \left\{i \in \mathbb{N}: s_{i} \in F\right\}$. Given a state $s \in S$ and a set of final states $F \subseteq S$ we say that a final state is reachable from $s_{0}$ if there is a run $r \in \operatorname{Runs}^{\mathcal{L}}\left(s_{0}\right)$ such that $\operatorname{Stop}(F)(r)<\infty$. A strategy of Player 1 is a partial function $\alpha: F R u n s^{\mathcal{L}} \rightarrow A$ such that for a run $r \in F R u n s^{\mathcal{L}}$ we have that $\alpha(r)$ is defined if last $(r) \in S_{1}$, and $\alpha(r) \in A($ last $(r))$ for every such $r$. A strategy of Player 2 is defined analogously. Let $\Sigma_{1}^{\mathcal{L}}$ and $\Sigma_{2}^{\mathcal{L}}$ be the set of strategies of Player 1 and Player 2, respectively. The unique run $\operatorname{Run}(s, \alpha, \tau)$ from a state $s$ when players use strategies $\alpha \in \Sigma_{1}^{\mathcal{L}}$ and $\tau \in \Sigma_{2}^{\mathcal{L}}$ is defined in a straightforward manner.

Given an initial state $s$ and a set of final states $F$, and strategies $\tau$ for Player 2 and $\alpha$ for Player 1 , Player 1 is said to win the reachability game if $\operatorname{Stop}(F)(\operatorname{Run}(s, \alpha, \tau))<\infty$, else if $\operatorname{Stop}(F)(\operatorname{Run}(s, \alpha, \tau))=$ $\infty$, then Player 2 is the winner. A reachability game problem is to decide whether in a given game arena $G$, an initial state $s$ and a set of final states $F$, Player 1 has a strategy to win the reachability game (irrespective of Player 2's strategy). 

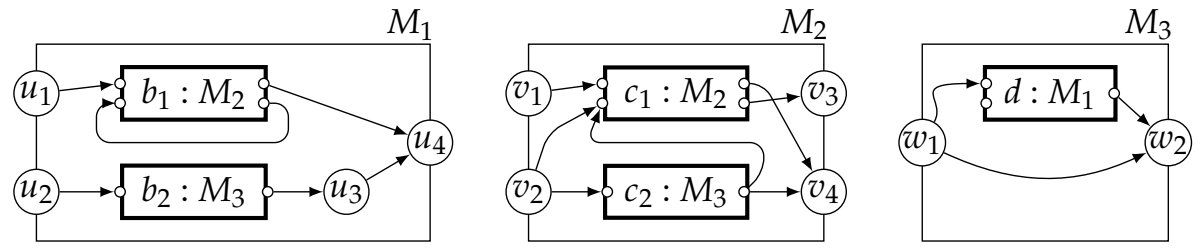

Figure 2: Example recursive state machine taken from [2]

\subsection{Reachability Games on Recursive state machines}

A recursive state machine [2] $\mathcal{M}$ is a tuple $\left(\mathcal{M}_{1}, \mathcal{M}_{2}, \ldots, \mathcal{M}_{k}\right)$ of components, where each component $\mathcal{M}_{i}=\left(N_{i}, \mathrm{EN}_{i}, \mathrm{EX}_{i}, B_{i}, Y_{i}, A_{i}, X_{i}\right)$ for each $1 \leq i \leq k$ is such that:

- $N_{i}$ is a finite set of nodes including a distinguished set $\mathrm{EN}_{i}$ of entry nodes and a set $\mathrm{EX}_{i}$ of exit nodes such that $\mathrm{EX}_{i}$ and $\mathrm{EN}_{i}$ are disjoint sets;

- $B_{i}$ is a finite set of boxes;

- $Y_{i}: B_{i} \rightarrow\{1,2, \ldots, k\}$ is a mapping that assigns every box to a component. We associate a set of call ports $\operatorname{Call}(b)$ and return ports $\operatorname{Ret}(b)$ to each box $b \in B_{i}$ :

$$
\text { Call }(b)=\left\{(b, e n): e n \in \operatorname{EN}_{Y_{i}(b)}\right\} \quad \text { and } \quad \operatorname{Ret}(b)=\left\{(b, e x): e x \in \operatorname{EX}_{Y_{i}(b)}\right\} .
$$

Let Call ${ }_{i}=\cup_{b \in B_{i}} \operatorname{Call}(b)$ and $\operatorname{Ret}_{i}=\cup_{b \in B_{i}} \operatorname{Ret}(b)$ be the set of call and return ports of component $\mathcal{M}_{i}$. We define the set of locations $Q_{i}$ of component $\mathcal{M}_{i}$ as the union of the set of nodes, call ports and return ports, i.e. $Q_{i}=N_{i} \cup$ Call $_{i} \cup$ Ret $_{i}$;

- $A_{i}$ is a finite set of actions; and

- $X_{i}: Q_{i} \times A_{i} \rightarrow Q_{i}$ is the transition function with a condition that call ports and exit nodes do not have any outgoing transitions.

For the sake of simplicity, we assume that the set of boxes $B_{1}, \ldots, B_{k}$ and set of nodes $N_{1}, N_{2}, \ldots, N_{k}$ are mutually disjoint. We use symbols $N, B, A, Q, X$, etc. to denote the union of the corresponding symbols over all components.

An example of a RSM is shown in Figure 2 (taken from [9]). An execution of a RSM begins at the entry node of some component and depending upon the sequence of input actions the state evolves naturally like a labelled transition system. However, when the execution reaches an entry port of a box, this box is stored on a stack of pending calls, and the execution continues naturally from the corresponding entry node of the component mapped to that box. When an exit node of a component is encountered, and if the stack of pending calls is empty then the run terminates; otherwise, it pops the box from the top of the stack and jumps to the exit port of the just popped box corresponding to the just reached exit of the component. We formalize the semantics of a RSM using a discrete LTS, whose states are pairs consisting of a sequence of boxes, called the context, mimicking the stack of pending calls and the current location. Let $\mathcal{M}=\left(\mathcal{M}_{1}, \mathcal{M}_{2}, \ldots, \mathcal{M}_{k}\right)$ be an RSM where the component $\mathcal{M}_{i}$ is $\left(N_{i}, E n_{i}, E x_{i}, B_{i}, Y_{i}, A_{i}, X_{i}\right)$. The semantics of $\mathcal{M}$ is the discrete labelled transition system $[[\mathcal{M}]]=\left(S_{\mathcal{M}}, A_{\mathcal{M}}, X_{\mathcal{M}}\right)$ where:

- $S_{\mathcal{M}} \subseteq B^{*} \times Q$ is the set of states;

- $A_{\mathcal{M}}=\cup_{i=1}^{k} A_{i}$ is the set of actions;

- $X_{\mathcal{M}}: S_{\mathcal{M}} \times A_{\mathcal{M}} \rightarrow S_{\mathcal{M}}$ is the transition function such that for $s=(\langle\kappa\rangle, q) \in S_{\mathcal{M}}$ and $a \in A_{\mathcal{M}}$, we have that $s^{\prime}=X_{\mathcal{M}}(s, a)$ if and only if one of the following holds:

1. the location $q$ is a call port, i.e. $q=(b, e n) \in$ Call, and $s^{\prime}=(\langle\kappa, b\rangle$,en); 
2. the location $q$ is an exit node, i.e. $q=e x \in \mathrm{EX}$ and $s^{\prime}=\left(\left\langle\kappa^{\prime}\right\rangle,(b, e x)\right)$ where $(b, e x) \in \operatorname{Ret}(b)$ and $\kappa=\left(\kappa^{\prime}, b\right)$;

3. the location $q$ is any other kind of location, and $s^{\prime}=\left(\langle\kappa\rangle, q^{\prime}\right)$ and $q^{\prime} \in X(q, a)$.

Given $\mathcal{M}$ and a subset $Q^{\prime} \subseteq Q$ of its nodes we define $\left[\left[Q^{\prime}\right]\right]_{\mathcal{M}}$ as $\left\{\left(\langle\kappa\rangle, v^{\prime}\right): \kappa \in B^{*}\right.$ and $\left.v^{\prime} \in Q^{\prime}\right\}$. We define the terminal configurations $\operatorname{Term}_{\mathcal{M}}$ as the set $\{(\langle\varepsilon\rangle, e x):$ ex $\in \mathrm{EX}\}$ with the empty context $\langle\varepsilon\rangle$. Given a recursive state machine $\mathcal{M}$, an initial node $v$, and a set of final locations $F \subseteq Q$ the reachability problem on $\mathcal{M}$ is defined as the reachability problem on the LTS [[M]] with the initial state $(\langle\varepsilon\rangle, v)$ and final states $[[F]]$. We define termination problem as the reachability of one of the exits with the empty context. The reachability and the termination problem for recursive state machines can be solved in polynomial time [2].

A partition $\left(Q_{1}, Q_{2}\right)$ of locations $Q$ of an RSM $\mathcal{M}$ (between Player 1 and Player 2) gives rise to recursive game arena $G=\left(\mathcal{M}, Q_{1}, Q_{2}\right)$. Given an initial state, $v$, and a set of final states, $F$, the reachability game on $\mathcal{M}$ is defined as the reachability game on the game arena $\left([[\mathcal{M}]],\left[\left[Q_{1}\right]\right]_{\mathcal{M}},\left[\left[Q_{2}\right]\right]_{\mathcal{M}}\right)$ with the initial state $(\langle\varepsilon\rangle, v)$ and the set of final states $[[F]]_{\mathcal{M}}$. Also, the termination game $\mathcal{M}$ is defined as the reachability game on the game arena $\left([[\mathcal{M}]],\left[\left[Q_{1}\right]\right]_{\mathcal{M}},\left[\left[Q_{2}\right]\right]_{\mathcal{M}}\right)$ with the initial state $(\langle\varepsilon\rangle, v)$ and the set of final states $\operatorname{Term}_{\mathcal{M}}$. It is a well known result (see, e.g. [10, 5]) that reachability games and termination games on RSMs are decidable (EXPTIME-complete).

\section{Recursive Hybrid Automata}

In this paper since we study both recursive timed automata as well as recursive stopwatch automata, we introduce a more general recurisve hybrid automata, and from that we define these two subclasses. Recursive hybrid automata (RHAs) extend classical hybrid automata (HAs) with recursion in a similar way RSMs extend LTSs. We introduce a rather simpler subclass of hybrid automata known as singular hybrid automata where all variables grow with constant-rates.

\subsection{Syntax}

Let $\mathbb{R}$ be the set of real numbers. Let $\mathcal{X}$ be a finite set of real-valued variables. A valuation on $\mathcal{X}$ is a function $v: \mathcal{X} \rightarrow \mathbb{R}$. We assume an arbitrary but fixed ordering on the variables and write $x_{i}$ for the variable with order $i$. This allows us to treat a valuation $v$ as a point $\left(v\left(x_{1}\right), v\left(x_{2}\right), \ldots, v\left(x_{n}\right)\right) \in \mathbb{R}^{|\mathcal{X}|}$. Abusing notations slightly, we use a valuation on $\mathcal{X}$ and a point in $\mathbb{R}^{|\mathcal{X}|}$ interchangeably. For a subset of variables $X \subseteq \mathcal{X}$ and a valuation $v^{\prime} \in \mathcal{X}$, we write $v\left[X:=v^{\prime}\right]$ for the valuation where $v\left[X:=v^{\prime}\right](x)=$ $v^{\prime}(x)$ if $x \in X$, and $v\left[X:=v^{\prime}\right](x)=v(x)$ otherwise. The valuation $\mathbf{0} \in \mathbb{R}^{|\mathcal{X}|}$ is a special valuation such that $\mathbf{0}(x)=0$ for all $x \in \mathcal{X}$.

We define a constraint over a set $\mathcal{X}$ as a subset of $\mathbb{R}^{|\mathcal{X}|}$. We say that a constraint is rectangular if it is defined as the conjunction of a finite set of constraints of the form $x \bowtie k$, where $k \in \mathbb{Z}, x \in \mathcal{X}$, and $\bowtie \in\{<, \leq,=,>, \geq\}$. For a constraint $G$, we write $[[G]]$ for the set of valuations in $\mathbb{R}^{|\mathcal{X}|}$ satisfying the constraint $G$. We write $\top$ ( resp., $\perp$ ) for the special constraint that is true (resp., false) in all the valuations, i.e. $[[\top]]=\mathbb{R}^{|\mathcal{X}|}$ (resp., $[[\perp]]=\varnothing$ ). We write $\operatorname{rect}(\mathcal{X})$ for the set of rectangular constraints over $\mathcal{X}$ including $T$ and $\perp$.

Definition 1 (Recursive Hybrid Automata) $A$ recursive hybrid automaton $\mathcal{H}=\left(\mathcal{X},\left(\mathcal{H}_{1}, \mathcal{H}_{2}, \ldots, \mathcal{H}_{k}\right)\right)$ is a pair made of a set of variables $\mathcal{X}$ and a collection of components $\left(\mathcal{H}_{1}, \mathcal{H}_{2}, \ldots, \mathcal{H}_{k}\right)$ where every component $\mathcal{H}_{i}=\left(N_{i}, \mathrm{EN}_{i}, \mathrm{EX}_{i}, B_{i}, Y_{i}, A_{i}, X_{i}, P_{i}, \operatorname{Inv}_{i}, E_{i}, J_{i}, F_{i}\right)$ is such that: 
- $N_{i}$ is a finite set of nodes including a distinguished set $\mathrm{EN}_{i}$ of entry nodes and a set $\mathrm{EX}_{i}$ of exit nodes such that $\mathrm{EX}_{i}$ and $\mathrm{EN}_{i}$ are disjoint sets;

- $B_{i}$ is a finite set of boxes;

- $Y_{i}: B_{i} \rightarrow\{1,2, \ldots, k\}$ is a mapping that assigns every box to a component. (Call ports Call(b) and return ports $\operatorname{Ret}(b)$ of a box $b \in B_{i}$, and call ports Call and $_{i}$ return ports Ret $_{i}$ of a component $\mathcal{H}_{i}$ are defined as before. We set $Q_{i}=N_{i} \cup$ Call $_{i} \cup$ Ret $_{i}$ and refer to this set as the set of locations of $\mathcal{H}_{i}$.)

- $A_{i}$ is a finite set of actions.

- $X_{i}: Q_{i} \times A_{i} \rightarrow Q_{i}$ is the transition function with a condition that call ports and exit nodes do not have any outgoing transitions.

- $P_{i}: B_{i} \rightarrow 2^{\mathcal{X}}$ is pass-by-value mapping that assigns every box the set of variables that are passed by value to the component mapped to the box; (The rest of the variables are assumed to be passed by reference.)

- $\operatorname{Inv}_{i}: Q_{i} \rightarrow \operatorname{rect}(\mathcal{X})$ is the invariant condition;

- $E_{i}: Q_{i} \times A_{i} \rightarrow \operatorname{rect}(\mathcal{X})$ is the action enabledness function;

- $J_{i}: A_{i} \rightarrow 2^{\mathcal{X}}$ is the variable reset function; and

- $F_{i}: Q_{i} \rightarrow \mathbb{N}^{|\mathcal{X}|}$ is the flow function characterizing the rate of each variable in each location.

We assume that the sets of boxes, nodes, locations, etc. are mutually disjoint across components and we write $(N, B, Y, Q, P, X$, etc.) to denote corresponding union over all components.

We say that a recursive hybrid automaton is glitch-free if for every box either all variables are passed by value or none is passed by value, i.e. for each $b \in B$ we have that either $P(b)=\mathcal{X}$ or $P(b)=\varnothing$. Any general recursive hybrid automaton with one variable is trivially glitch-free. We say that a RHA is hierarchical if there exists an ordering over components such that a component never invokes another component of higher order or same order.

We say that a variable $x \in \mathcal{X}$ is a clock (resp., a stopwatch) if for every location $q \in Q$ we have that $F(q)(x)=1$ (resp., $F(q)(x) \in\{0,1\}$ ). A recursive timed automaton (RTA) is simply a recursive hybrid automata where all variables $x \in \mathcal{X}$ are clocks. Similarly, we define a recursive stopwatch automaton (RSA) as a recursive hybrid automaton where all variables $x \in \mathcal{X}$ are stopwatches. Since all of our results pertaining to recursive hybrid automata are shown in the context of recursive stopwatch automata, we often confuse RHA with RSA.

\subsection{Semantics}

A configuration of an RHA $\mathcal{H}$ is a tuple $(\langle\kappa\rangle, q, v)$, where $\kappa \in\left(B \times \mathbb{R}^{|\mathcal{X}|}\right)^{*}$ is sequence of pairs of boxes and variable valuations, $q \in Q$ is a location and $v \in \mathbb{R}^{|\mathcal{X}|}$ is a variable valuation over $\mathcal{X}$ such that $v \in$ $\operatorname{Inv}(q)$. The sequence $\langle\kappa\rangle \in\left(B \times \mathbb{R}^{|\mathcal{X}|}\right)^{*}$ denotes the stack of pending recursive calls and the valuation of all the variables at the moment that call was made, and we refer to this sequence as the context of the configuration. Technically, it suffices to store the valuation of variables passed by value, because other variables retain their value after returning from a call to a box, but storing all of them simplifies the notation. We denote the empty context by $\langle\epsilon\rangle$. For any $t \in \mathbb{R}$, we let $(\langle\kappa\rangle, q, v)+t$ equal the configuration $(\langle\kappa\rangle, q, v+F(q) \cdot t)$. Informally, the behaviour of an RHA is as follows. In configuration $(\langle\kappa\rangle, q, v)$ time passes before an available action is triggered, after which a discrete transition occurs. Time passage is 
available only if the invariant condition $\operatorname{Inv}(q)$ is satisfied while time elapses, and an action $a$ can be chosen after time $t$ elapses only if it is enabled after time elapse, i.e., if $v+F(q) \cdot t \in E(q, a)$. If the action $a$ is chosen then the successor state is $\left(\langle\kappa\rangle, q^{\prime}, v^{\prime}\right)$ where $q^{\prime} \in X(q, a)$ and $v^{\prime}=(v+t)[J(a):=\mathbf{0}]$. Formally, the semantics of an RHA is given by an LTS which has both an uncountably infinite number of states and transitions.

Definition 2 (RHA semantics) Let $\mathcal{H}=\left(\mathcal{X},\left(\mathcal{H}_{1}, \mathcal{H}_{2}, \ldots, \mathcal{H}_{k}\right)\right)$ be an $R H A$ where each component is of the form $\mathcal{H}_{i}=\left(N_{i}, \mathrm{EN}_{i}, \mathrm{EX}_{i}, B_{i}, Y_{i}, A_{i}, X_{i}, P_{i}, I n v_{i}, E_{i}, J_{i}, F_{i}\right)$. The semantics of $\mathcal{H}$ is a labelled transition system $[[\mathcal{H}]]=\left(S_{\mathcal{H}}, A_{\mathcal{H}}, X_{\mathcal{H}}\right)$ where:

- $S_{\mathcal{H}} \subseteq\left(B \times \mathbb{R}^{|\mathcal{X}|}\right)^{*} \times Q \times \mathbb{R}^{|\mathcal{X}|}$, the set of states, is s.t. $(\langle\kappa\rangle, q, v) \in S_{\mathcal{H}}$ if $v \in \operatorname{Inv}(q)$.

- $A_{\mathcal{H}}=\mathbb{R}_{\oplus} \times A$ is the set of timed actions, where $\mathbb{R}_{\oplus}$ is the set of non-negative reals;

- $X_{\mathcal{H}}: S_{\mathcal{H}} \times A_{\mathcal{H}} \rightarrow S_{\mathcal{H}}$ is the transition function such that for $(\langle\kappa\rangle, q, v) \in S_{\mathcal{H}}$ and $(t, a) \in A_{\mathcal{H}}$, we have $\left(\left\langle\kappa^{\prime}\right\rangle, q^{\prime}, v^{\prime}\right)=X_{\mathcal{H}}((\langle\kappa\rangle, q, v),(t, a))$ if and only if the following condition holds:

1. if the location $q$ is a call port, i.e. $q=(b, e n) \in$ Call then $t=0$, the context $\left\langle\kappa^{\prime}\right\rangle=\langle\kappa,(b, v)\rangle$, $q^{\prime}=e n$, and $v^{\prime}=v$.

2. if the location $q$ is an exit node, i.e. $q=e x \in E x,\langle\kappa\rangle=\left\langle\kappa^{\prime \prime},\left(b, v^{\prime \prime}\right)\right\rangle$, and let $(b, e x) \in \operatorname{Ret}(b)$, then $t=0 ;\left\langle\kappa^{\prime}\right\rangle=\left\langle\kappa^{\prime \prime}\right\rangle ; q^{\prime}=(b, e x) ;$ and $v^{\prime}=v\left[P(b):=v^{\prime \prime}\right]$.

3. if location $q$ is any other kind of location, then $\left\langle\kappa^{\prime}\right\rangle=\langle\kappa\rangle, q^{\prime} \in X(q, a)$, and

(a) $v+F(q) \cdot t^{\prime} \in \operatorname{Inv}(q)$ for all $t^{\prime} \in[0, t]$;

(b) $v+F(q) \cdot t \in E(q, a)$;

(c) $v^{\prime}=(v+F(q) \cdot t)[J(a):=\mathbf{0}]$.

\subsection{Reachability and Time-Bounded Reachability Game Problems}

For a subset $Q^{\prime} \subseteq Q$ of states of RHA $\mathcal{H}$ we define the set $\left[\left[Q^{\prime}\right]\right]_{\mathcal{H}}$ as the set $\left\{(\langle\kappa\rangle, q, v) \in S_{\mathcal{H}}: q \in Q^{\prime}\right\}$. We define the terminal configurations as $\operatorname{Term}_{\mathcal{H}}=\left\{(\langle\varepsilon\rangle, q, v) \in S_{\mathcal{H}}: q \in \mathrm{EX}\right\}$. Given a recursive hybrid automaton $\mathcal{H}$, an initial node $q$ and valuation $v \in \mathbb{R}^{|\mathcal{X}|}$, and a set of final locations $F \subseteq Q$, the reachability problem on $\mathcal{H}$ is to decide the existence of a run in the LTS $[[\mathcal{H}]]$ staring from the initial state $(\langle\varepsilon\rangle, q, v)$ to some state in $[[F]]_{\mathcal{H}}$. As with RSMs, we also define termination problem as reachability of one of the exits with the empty context. Hence, given an RHA $\mathcal{H}$ and an initial node $q$ and a valuation $v \in \mathbb{R}^{|\mathcal{X}|}$, the termination problem on $\mathcal{H}$ is to decide the existence of a run in the LTS $[[\mathcal{H}]]$ from initial state $(\langle\varepsilon\rangle, q, v)$ to a final state in $\operatorname{Term}_{\mathcal{H}}$.

Given a run $r=\left\langle s_{0},\left(t_{1}, a_{1}\right), s_{2},\left(t_{2}, a_{2}\right), \ldots,\left(s_{n}, t_{n}\right)\right\rangle$ of an RHA, its time duration time $(r)$ is defined as $\sum_{i=1}^{n} t_{i}$. Given a recursive hybrid automaton $\mathcal{H}$, an initial node $q$, a bound $T \in \mathbb{N}$, and valuation $v \in \mathbb{R}^{|\mathcal{X}|}$, and a set of final locations $F \subseteq Q$, the time-bounded reachability problem on $\mathcal{H}$ is to decide the existence of a run $r$ in the LTS $[[\mathcal{H}]]$ staring from the initial state $(\langle\varepsilon\rangle, q, v)$ to some state in $[[F]]_{\mathcal{H}}$ such that time $(r) \leq T$. Time-bounded termination problem is defined in an analogous manner.

A partition $\left(Q_{1}, Q_{2}\right)$ of locations $Q$ of an RHA $\mathcal{H}$ gives rise to a recursive hybrid game arena $\Gamma=\left(\mathcal{H}, Q_{1}, Q_{2}\right)$. Given an initial location $q$, a valuation $v \in V$ and a set of final states $F$, the reachability game on $\Gamma$ is defined as the reachability game on the game arena $\left([[\mathcal{H}]],\left[\left[Q_{1}\right]\right]_{\mathcal{H}},\left[\left[Q_{2}\right]\right]_{\mathcal{H}}\right)$ with the initial state $(\langle\varepsilon\rangle,(q, v))$ and the set of final states $[[F]]_{\mathcal{H}}$. Also, termination game on $\Gamma$ is defined as the reachability game on the game arena $\left([[\mathcal{H}]],\left[\left[Q_{1}\right]\right]_{\mathcal{H}},\left[\left[Q_{2}\right]\right]_{\mathcal{H}}\right)$ with the initial state $(\langle\varepsilon\rangle,(q, v))$ and the set of final states $\operatorname{Term}_{\mathcal{H}}$.

We prove the following key theorem about reachability games on various subclasses of recursive hybrid automata in Section 4 
Theorem 1 The reachability game problem is undecidable for:

1. Unrestricted RSA with 2 stopwatches,

2. Glitch-free RSA with 3 stopwatches,

3. Unrestricted RTA with 3 clocks under bounded time, and

4. Glitch-free RSA with 4 stopwatches under bounded time.

Moreover, all of these results hold even under hierarchical restriction.

On a positive side, we observe that for glitch-free RSA with two stopwatches reachability games are decidable by exploiting the existence of finite bisimulation for hybrid automata with 2 stopwatches. Details can be found in [6].

Theorem 2 The reachability games are decidable for glitch-free RSA with atmost two stopwatches.

\section{Undecidability Results}

In this section, we provide a proof sketch of our undecidability results by reducing the halting problem for two counter machines to the reachability problem in an RHA/RTA. Before we show our reduction, we give a formal definition of two-counter machines.

Definition 3 (Two-counter Machines) A two-counter machine is a tuple $(L, C)$ where $L=\left\{\ell_{0}, \ell_{1}, \ldots, \ell_{n}\right\}$ is the set of instructions-including a distinguished terminal instruction $\ell_{n}$ called HALT-and $C=\left\{c_{1}, c_{2}\right\}$ is the set of two counters. The instructions L are of the type:

1. (increment c) $\ell_{i}: c:=c+1$; goto $\ell_{k}$,

2. (decrement $c) \ell_{i}: c:=c-1$; goto $\ell_{k}$,

3. (zero-check $c) \ell_{i}$ : if $(c>0)$ then goto $\ell_{k}$ else goto $\ell_{m}$,

4. (Halt) $\ell_{n}: H A L T$.

where $c \in C, \ell_{i}, \ell_{k}, \ell_{m} \in L$.

A configuration of a two-counter machine is a tuple $(l, c, d)$ where $l \in L$ is an instruction, and $c, d$ are natural numbers that specify the value of counters $c_{1}$ and $c_{2}$, respectively. The initial configuration is $\left(\ell_{0}, 0,0\right)$. A run of a two-counter machine is a (finite or infinite) sequence of configurations $\left\langle k_{0}, k_{1}, \ldots\right\rangle$ where $k_{0}$ is the initial configuration, and the relation between subsequent configurations is governed by transitions between respective instructions. The run is a finite sequence if and only if the last configuration is the terminal instruction $\ell_{n}$. Note that a two-counter machine has exactly one run starting from the initial configuration. The halting problem for a two-counter machine asks whether its unique run ends at the terminal instruction $\ell_{n}$. The halting problem [7] for two-counter machines is undecidable.

For all the undecidability results, we construct a recursive automaton (timed/hybrid) as per the case, whose main components are the modules for the instructions and the counters are encoded in the variables of the automaton. In these reductions, the reachability of the exit node of each component corresponding to an instruction is linked to a faithful simulation of various increment, decrement and zero check instructions of the machine by choosing appropriate delays to adjust the clocks/variables, to reflect changes in counter values. We specify a main component for each type instruction of the two counter machine, for example $\mathcal{H}_{\text {inc }}$ for increment. The entry node and exit node of a main component $\mathcal{H}_{\text {inc }}$ corresponding to 
an instruction $\left[\ell_{i}: c:=c+1\right.$; goto $\left.\ell_{k}\right]$ are respectively $\ell_{i}$ and $\ell_{k}$. Similarly, a main component corresponding to a zero check instruction $\left[l_{i}\right.$ : if $(c>0)$ then goto $\left.\ell_{k}\right]$ else goto $\ell_{m}$, has a unique entry node $\ell_{i}$, and two exit nodes corresponding to $\ell_{k}$ and $\ell_{m}$ respectively. The various main components corresponding to the various instructions, when connected appropriately, gives the higher level component $\mathcal{H}_{M}$ and this completes the RHA $\mathcal{H}$. The entry node of $\mathcal{H}_{M}$ is the entry node of the main component for the first instruction of $M$ and the exit node is $H A L T$. Player 1 simulates the machine while Player 2 verifies the simulation. Suppose in each main component for each type of instruction correctly Player 1 simulates the instruction by accurately updating the counters encoded in the variables of $\mathcal{H}$. Then, the unique run in $M$ corresponds to an unique run in $\mathcal{H}_{M}$. The halting problem of the two counter machine now boils down to existence of a Player 1 strategy to ensure the reachability of an exit node HALT (and $\ddot{*}$ ) in $\mathcal{H}_{M} ! 1$

For the correctness proofs, we represent runs in the RSA using three different forms of transitions $s \underset{t}{\stackrel{g, J}{\longrightarrow}} s^{\prime}, s \rightsquigarrow s^{\prime}$ and $s \underset{M(V)}{\stackrel{*}{\longrightarrow}} s^{\prime}$ defined in the following way:

1. The transitions of the form $s \underset{t}{\stackrel{g_{\prime} J}{\longrightarrow}} s^{\prime}$, where $s=(\langle\kappa\rangle, n, v), s^{\prime}=\left(\langle\kappa\rangle, n^{\prime}, v^{\prime}\right)$ are configurations of the RHA, $g$ is a constraint or guard on variables that enables the transition, $J$ is a set of variables, and $t$ is a real number, holds if there is a transition in the RHA from vertex $n$ to $n^{\prime}$ with guard $g$ and reset set $J$. Also, $v^{\prime}=v+r t[J:=0]$, where $r$ is the rate vector of state $s$.

2. The transitions of the form $s \rightsquigarrow s^{\prime}$ where $s=(\langle\kappa\rangle, n, v), s^{\prime}=\left(\left\langle\kappa^{\prime}\right\rangle, n^{\prime}, v^{\prime}\right)$ correspond to the following cases:

- transitions from a call port to an entry node. That is, $n=(b, e n)$ for some box $b \in B$ and $\kappa^{\prime}=\langle\kappa,(b, v)\rangle$ and $n^{\prime}=e n \in \mathrm{EN}$ while $v^{\prime}=v$.

- transitions from an exit node to a return port which restores values of the variables passed by value, that is, $\langle\kappa\rangle=\left\langle\kappa^{\prime \prime},\left(b, v^{\prime \prime}\right)\right\rangle, n=e x \in \mathrm{EX}$ and $n^{\prime}=(b, e x) \in \operatorname{Ret}(b)$ and $\kappa^{\prime}=\kappa^{\prime \prime}$, while $v^{\prime}=v\left[P(b):=v^{\prime \prime}\right]$.

3. The transitions of the form $s \underset{M(V)}{\stackrel{t}{\longrightarrow}} s^{\prime}$, called summary edges, where $s=(\langle\kappa\rangle, n, v), s^{\prime}=\left(\langle\kappa\rangle, n^{\prime}, v^{\prime}\right)$ are such that $n=(b, e n)$ and $n^{\prime}=(b, e x)$ are call and return ports, respectively, of a box $b$ mapped to $M$ which passes by value to $M$, the variables in $V . t$ is the time elapsed between the occurences of $(b, e n)$ and $(b, e x)$. In other words, $t$ is the time elapsed in the component $M$.

A configuration $(\langle\kappa\rangle, n, v)$ is also written as $(\langle\kappa\rangle, n,(v(x), v(y)))$.

\subsection{Time Bounded Reachability Games in Unrestricted RTA}

Lemma 1 The time bounded reachability game problem is undecidable for recursive timed automata with at least 3 clocks.

Proof. We prove that the reachability problem is undecidable for unrestricted RTA with 3 clocks. In order to obtain the undecidability result, we use a reduction from the halting problem for two counter machines. Our reduction uses a RTA with three clocks $x, y, z$.

We specify a main component for each instruction of the two counter machine. On entry into a main component for increment/decrement/zero check, we have $x=\frac{1}{2^{k+c} 3^{k+d}}, y=\frac{1}{2^{k}}$ and $z=0$, where $c, d$ are the current values of the counters and $k$ is the current instruction. Note that $z$ is used only to enforce

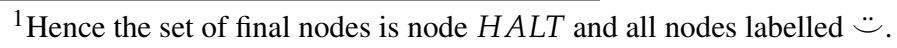


urgency in several locations. Given a two counter machine, we build a 3 clock RTA whose building blocks are the main components for the instructions. The purpose of the components is to simulate faithfully the counter machine by choosing appropriate delays to adjust the variables to reflect changes in counter values. On entering the entry node $e n$ of a main component corresponding to an instruction $l_{i}$, we have the configuration $\left(\langle\epsilon\rangle, e n,\left(\frac{1}{2^{k+c} 3^{k+d}}, \frac{1}{2^{k}}, 0\right)\right)$ of the three clock RTA.

We discuss the module for incrementing counter $c$ here; more details can be found in [6]. In all the components, the variables passed by value are written below the boxes and the invariants of the locations are indicated below them.

Simulate increment instruction: Lets consider the increment instruction $\ell_{i}: c=c+1$; goto $\ell_{k}$. The component for this instruction is component Inc $c$ given in Figure 3. Assume that $x=\frac{1}{2^{k+c} 3^{k+d}}, y=\frac{1}{2^{k}}$ and $z=0$ at the entry node $e n_{1}$ of the component $I n c c$. To correctly simulate the increment of counter $c$, the clock values at the exit node $e x_{1}$ should be $x=\frac{1}{2^{k+c+2} 3^{k+d+1}}, y=\frac{1}{2^{k+1}}$ and $z=0$. The value of $x$ goes from $x=\frac{1}{2^{k+c} 3^{k+d}}$ to $x=\frac{1}{2^{k+c+2} 3^{k+d+1}}$ so that $c$ is incremented and end of current $k+1$ instruction is also recorded. Thus $x=\frac{1}{2^{k+1+c+1} 3^{k+1+d}}$.

Let $\alpha=\frac{1}{2^{k+c} 3^{k+d}}$ and $\beta=\frac{1}{2^{k}}$. We want $x=\frac{\alpha}{12}$ and $y=\frac{\beta}{2}$ at $e x_{1}$. We utilise the component $\operatorname{Div}\{a, n\}$ (instantiating $\operatorname{Div}\{a, n\}$ with $a=x, n=12$ to achieve $x=\frac{\alpha}{12}$ and with $a=y, n=2$ to achieve $y=\frac{\beta}{2}$ ) to perform these divisions. Lets walk through the working of the component Inc c. As seen above, at the entry node $e n_{1}$, we have $x=\frac{1}{2^{k+c} 3^{k+d}}, y=\frac{1}{2^{k}}$ and $z=0$.

1. No time is spent at $e n_{1}$ due to the invariant $z=0$. $\operatorname{Div}\{y, 2\}$ is called, passing $x, z$ by value. At the call port of $A_{1}: \operatorname{Div}\{y, 2\}$, we have the same values of $x, y, z$. Let us examine the component $\operatorname{Div}\{y, 2\}$. We instantiate $\operatorname{Div}\{a, n\}$ with $a=y, n=2$. Thus, the clock referred to as $b$ in $\operatorname{Div}\{a, n\}$ is $x$ after the instantiation. At the entry node $e n_{2}$ of $\operatorname{Div}\{y, 2\}$, no time is spent due to the invariant $z=0$; we have $a=y=\beta, b=x=\alpha, z=0$. Resetting $b$ (i.e; $x$ ), we are at the call port of $A_{3}: D . A_{3}$ is called, passing $a, z$ by value. A nondeterministic time $t$ is spent at the entry node $e n_{3}$ of $D$. Thus, at the return port of $A_{3}$, we have $a=y=\beta, b=x=t, z=0$. The return port of $A_{3}$ is a node belonging to Player 2; for Player 1 to reach $\ddot{*}, t$ must be $\frac{\beta}{2}$. Player 2 has two choices to make at the return port of $A_{3}$ : he can continue the simulation, by resetting $a($ i.e; $y)$ and going to the call port of $A_{5}: D$, or he can verify if $t$ is indeed $\frac{\beta}{2}$, by going to the call port of $A_{4}$.

- Assume Player 2 goes to the call port of $A_{4}: C_{y / 2}^{x=}$ (recall, that by the instantiation, $b=x$, $a=y$ and $n=2$ ). $z$ is passed by value. At the entry node $e n_{5}$ of $C_{y / 2}^{x=}$, no time elapses due to the invariant $z=0$. Thus, we have $x=b=t, a=y=\beta, z=0$ at $e n_{5}$. The component $A_{7}: M_{x}$ is invoked, passing $x, z$ by value. At the entry node $e n_{6}$ of $M_{b}$, a time $1-t$ is spent, giving $a=y=\beta+1-t, b=x=t$ and $z=0$ at the return port of $A_{7}$. Since $n=2$, one more invocation of $A_{7}: M_{x}$ is made, obtaining $a=y=\beta+2(1-t), b=x=t$ and $z=0$ at the return port of $A_{7}$ after the second invocation. To reach the exit node $e x_{5}$ of $C_{y / 2}^{x=}, a$ must be exactly 2 , since no time can be spent at the return port of $A_{7}$; this is so since the invariant $z=0$ at the exit node $e x_{5}$ of $C_{y / 2}^{x=}$ is satisfied only when no time is spent at the return port of $A_{7}$. If $a$ is exactly 2 , we have $\beta=2 t$. In this case, from the return port of $A_{4}, \ddot{*}$ can be reached.

- Now consider the case that Player 2 moves ahead from the return port of $A_{3}$, resetting a(i.e; $y)$ to the call port of $A_{5}: D$. The values are $a=y=0, b=x=t=\frac{\beta}{2}$ and $z=0 . A_{5}: D$ is invoked passing $b=x$ and $z$ by value. A non-deterministic amount of time $t^{\prime}$ is spent at the entry node $e n_{3}$ of $D$, giving $a=y=t^{\prime}, b=x=\frac{\beta}{2}$ and $z=0$ at the return port of $A_{5}$. Again, 


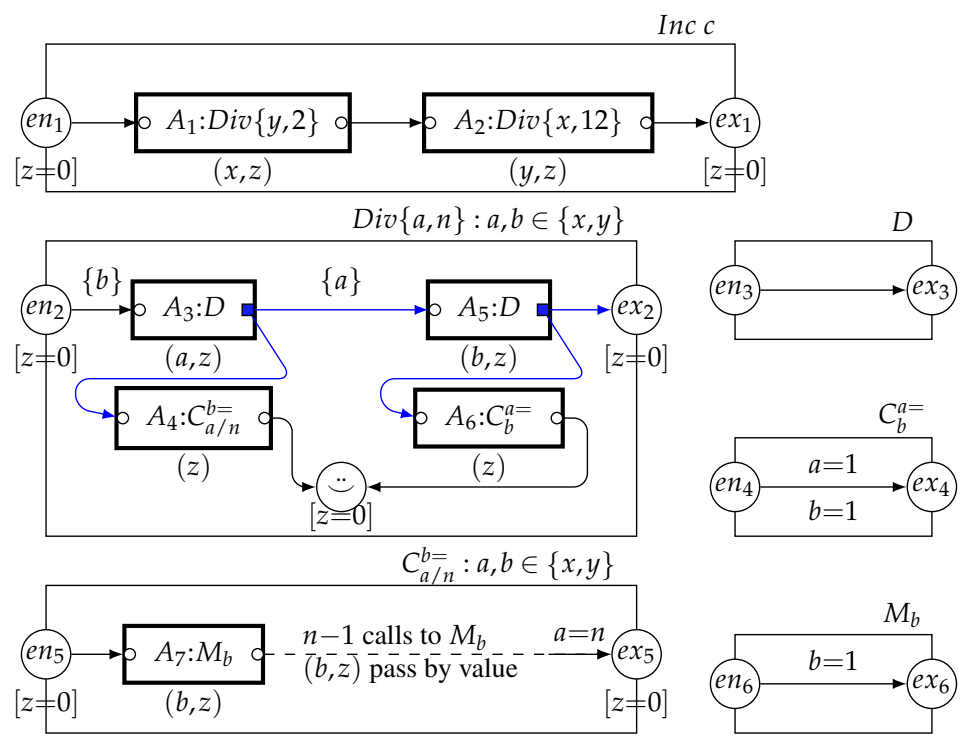

Figure 3: Games on RTA with 3 clocks : Increment $c$.

the return port of $A_{5}$ is a node belonging to Player 2. Here Player 2, thus has two choices: he can continue with the simulation going to $e x_{2}$, or can verify that $t^{\prime}=\frac{\beta}{2}$ by going to the call port of $A_{6}: C_{x}^{y=} \cdot C_{x}^{y=}$ is a component that checks if $y$ has "caught up" with $x$; that is, whether $t^{\prime}=t=\frac{\beta}{2}$. At the entry node $e n_{4}$ of $C_{x}^{y=}, a$ and $b$ can simultaneeously reach 1 iff $t=t^{\prime}$; that is, $t^{\prime}=\frac{\beta}{2}$. Then, from the return port of $A_{6}$, we can reach $\ddot{*}$.

- Thus, we reach $e x_{2}$ with $x=y=\frac{\beta}{2}, z=0$. At the return port of $A_{1}: \operatorname{Div}\{y, 2\}$, we thus have $x=\alpha, y=\frac{\beta}{2}, z=0$.

2. From the return port of $A_{1}: \operatorname{Div}\{y, 2\}$, we reach the call port of $A_{2}: \operatorname{Div}\{x, 12\} \cdot y, z$ are passed by value. The functioning of $A_{2}$ is similar to that of $A_{1}$ : at the return port of $A_{1}$, we obtain $x=\frac{\alpha}{12}$, $y=\frac{\beta}{2}$ and $z=0$.

Time taken: Now we discuss the total time to reach a $\ddot{*}$ node or the exit node $e x_{1}$ of the component Inc $c$ while simulating the increment instruction. At the entry node $e n_{1}$, clock values are $x=\frac{1}{2^{k+c} 3^{k+d}}$, $y=\frac{1}{2^{k}}$ and $z=0$. Let $\alpha=\frac{1}{2^{k+c 3^{k+d}}}$ and $\beta=\frac{1}{2^{k}}$. The invaraint $z=0$ at the entry and the exit nodes $e n_{1}$ and $e x_{1}$ ensures that no time elapses in these nodes and also in the return ports of $A_{1}$ and $A_{2}$. From the analysis above, it follows that at the return port of $A_{1}: \operatorname{Div}\{y, 2\}, x=\alpha, y=\frac{\beta}{2}$ and $z=0$. Similarly at the return port of $A_{2}: \operatorname{Div}\{x, 12\}$, the clock values are $x=\frac{\alpha}{12}, y=\frac{\beta}{2}$ and $z=0$. Thus, counter $c$ has been incremented and the end of instruction $k$ has been recorded in $x$ and $y$. The time spent along the path from $e n_{1}$ to $e x_{1}$ is the sum of times spent in $A_{1}: \operatorname{Div}\{y, 2\}$ and $A_{2}: \operatorname{Div}\{x, 12\}$.

- Time spent in $A_{1}: \operatorname{Div}\{y, 2\}$. The time spent in $A_{3}: D$, as well as $A_{5}: D$ is both $\frac{\beta}{2}$. Recall that Player 2 can verify that the times $t, t^{\prime}$ spent in $A_{3}, A_{5}$ are both $\frac{\beta}{2}$. If Player 2 enters $A_{4}$ to verify $t=\frac{\beta}{2}$, then the time taken is $2(1-t)$. In this case, the time taken to reach $\ddot{*}$ from the return port of $A_{4}$ is $t+2(1-t)=2-\frac{\beta}{2}$. Likewise, if Player 2 continued from $A_{3}$ to $A_{5}$, and goes on to verify that the time $t^{\prime}$ spent in $A_{5}$ is also $\frac{\beta}{2}$, then the total time spent before reaching the $\ddot{*}$ from 
the return port of $A_{6}$ is $t+t^{\prime}+\left(1-t^{\prime}\right)=1+t=1+\frac{\beta}{2}$. Thus, if we are back at the return port of $A_{1}$, the time spent in $A_{1}$ is $t+t^{\prime}=\beta$.

- Time spent in $A_{2}: \operatorname{Div}\{x, 12\}$. Here, the time spent in $A_{3}: D$ as well as $A_{5}: D$ is $\frac{\alpha}{12}$. In case Player 2 verifies that the time $t$ spent in $A_{3}: D$ is indeed $\frac{\alpha}{12}$, then he invokes $A_{4}$. The time elapsed in $C_{x / 12}^{y=}$ is $12(1-t)=12\left(1-\frac{\alpha}{12}\right)<12$. Likewise, if Player 2 continued from $A_{3}$ to $A_{5}$, and goes on to verify that the time $t^{\prime}$ spent in $A_{5}$ is also $\frac{\alpha}{12}$, then the total time spent before reaching

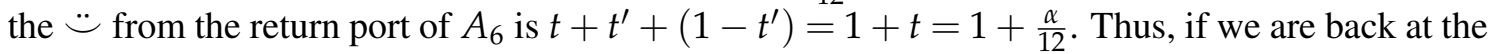
return port of $A_{2}$, the time spent in $A_{2}$ is $t+t^{\prime}=\frac{2 \alpha}{12}$.

- In general, the component $\operatorname{Div}\{a, n\}$ divides the value in clock $a$ by $n$. If $a=\zeta$ on entering $\operatorname{Div}\{a, n\}$, then upon exit, its value is $a=\frac{\zeta}{n}$. The time taken to reach the exit $e x_{2}$ is $2 *\left(\frac{\zeta}{n}\right)$. The time taken to reach the node $\ddot{*}$ in $\operatorname{Div}\{a, n\}$ is $<n$ (due to $n$ calls to $M_{b}$ component).

- Total time spent in Inc c. Thus, if we come back to the return port of $A_{2}$, the total time spent is $\beta+\frac{2 \alpha}{12}<2 \beta$, on entering with $y=\beta$. Recall that $x=\alpha=\frac{1}{2^{k+c 3^{k+d}}}$ and $y=\beta=\frac{1}{2^{k}}$ and thus $\alpha \leq \beta$ always.

In the zero check instruction, starting with $x=\frac{1}{2^{k+c} 3^{k+d}}=\alpha, y=\frac{1}{2^{k}}=\beta$ and $z=0$, we divide $x$ by 6 and $y$ by 2 , to record the $(k+1)$ th instruction. [6] gives the details. As in the case of the increment instruction, we show that the main module for simulation of a zero check instruction also takes a time $<2 \beta$, on entering with $y=\beta$. The module for the decrement instruction for counter $c$ only differs from the Increment $c$ module of Figure 3 , in that the call to $\operatorname{Div}\{x, 12\}$ is replaced by $\operatorname{Div}\{x, 3\}$ thus updating $x$ from $\frac{1}{2^{k+c} 3^{k+d}}$ to $\frac{1}{2^{k+c} 3^{k+d+1}}$. Similar is the case of incrementing and decrementing counter $d$.

We obtain the full RTA simulating the two counter machine by connecting the entry and exit of main components of instructions according to the machine's sequence of instructions. If the machine halts, then the RTA has an exit node corresponding to HALT. Establising that for the $k$ th instruction, the time elapsed is no more than $2 \beta$, for $\beta=\frac{1}{2^{k}}$, we have that for the first instruction, the time elapsed is at most 2 , for the second instruction it is $\frac{2}{2}$, for the third it is $\frac{2}{2^{2}}$ and so on. It is straightforward to see that the total time duration is bounded from above by $2\left(1+\frac{1}{2}+\frac{1}{4}+\frac{1}{8}+\frac{1}{16}+\cdots\right)<4$.

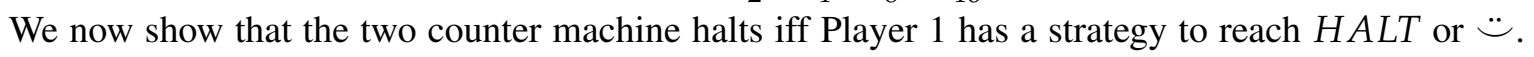
Suppose the machine halts. Then the strategy for Player 1 is to choose the appropriate delays to update the counters in each main component. Now if Player 2 does not verify (by entering check components) in any of the main components, then the exit $e x_{1}$ of the main component is reached. If Player 2 decides to verify then the node $\because$ is reached. Thus, if Player 1 simulates the machine correctly then either the HALT exit or $\ddot{*}$ is reached if the machine halts.

Conversely, assume that the two counter machine does not halt. Then we show that Player 1 has no strategy to reach either HALT or $\ddot{*}$. Consider a strategy of Player 1 which correctly simulates all the instructions. Then $\ddot{*}$ is reached only if Player 2 chooses to verify. But if Player 2 does not choose to verify then $\ddot{*}$ can not be reached. The simulation continues and as the machine does not halt, the exit node HALT is never reached. Now, consider any other strategy of Player 1 which does an error in simulation (in a hope to reach $H A L T$ ). Player 2 could verify this, and in this case, the node $\ddot{*}$ will not be reached as the delays are incorrect. Thus Player 1 can not ensure reaching HALT or $\ddot{*}$ with a simulation error. This is because there exists a strategy of Player 2 which can check the error and Player 1 thus can not win irrespective of all strategies of Player 2. 


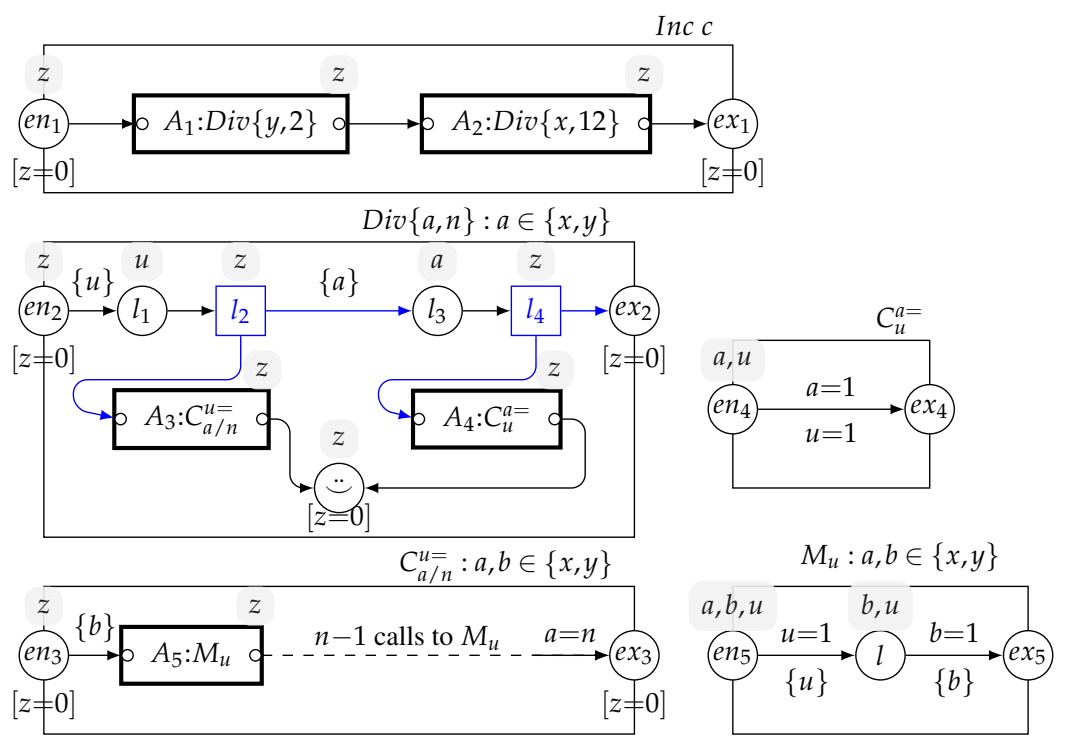

Figure 4: Games on Glitchfree-RSA with 4 stopwatches : Increment $c$. Note that the variables that tick in a location are indicated above it. Due to semantics of RSA, no time elapses in the call ports and exit nodes and hence variable-ticking is not mentioned for these locations.

\subsection{Time Bounded Reachability Games in RSA}

Lemma 2 The time bounded reachability game problem is undecidable for glitch-free recursive stopwatch automata with at least 4 stopwatches.

Proof. We outline quickly the changes as compared to Lemma 1 for the case of the increment instruction. Figure 4 gives the component for incrementing counter $c$. There are 4 stopwatches $x, y, z, u$. The encoding of the counters in the variables is similar to Lemma 1; at the entry node of each main component simulating the $k$ th instruction, we have $x=\frac{1}{2^{c+k} 3^{d+k}}=\alpha, y=\frac{1}{2^{k}}=\beta$ and $z=0$, where $c, d$ are the current values of the counters. We use the extra stopwatch $u$ for rough work and hence we do not ensure that $u=0$ when a component is entered.

As was the case in Lemma 1, simulation of the $(k+1)$ th instruction, incrementing $c$ amounts to dividing $y$ by 2 and $x$ by 12. In Lemma 1 , it was possible to pass some clocks by value, and some by reference, but here, all variables must be either passed by value or by reference. The $\operatorname{Div}\{a, n\}$ module here is similar to that in Lemma 1: the box $\left[A_{3}: D\right]$ in Figure 3 is replaced by the node $l_{1}$, where only $u$ ticks and accumulates a time $t$. (Recall that $u$ is the stopwatch used for rough work and has no bearing on the encoding.) In node $l_{2}$, only $z$ ticks. $l_{2}$ is a node belonging to Player 2 . The time $t$ spent at $l_{1}$ must be exactly $t=\frac{\beta}{2}$, where $\beta=\frac{1}{2^{k}}$ is the value of $a=y$ on entering Div $\{y, 2\}$. When $t=\frac{\beta}{2}$, Player 1 can reach $\ddot{*}$ even when Player 2 enters the check module $C_{y / 2}^{u=}$. Again, note that the module $C_{y / 2}^{u=}$ is similar to the one in Figure 3. We use the clock $x$ (instantiating $b=x$ ) for rough work in this component. Due to this, the earlier value of $x$ is lost. However, this does not affect the machine simulation as we reach the node $\ddot{*}$, and the simulation does not continue. $C_{y / 2}^{u=}$ calls the component $M_{u}$ : at the entry node $e n_{5}$ (of $M_{u}$ ), we have $b=x=0, u=t$ and $a=y=\beta . a, b, u$ tick at $e n_{5}$. A time $1-t$ is spent at $e n_{5}$, obtaining $b=1-t, u=0, a=\beta+(1-t)$ at $l$. At $l$, only $b, u$ tick obtaining $b=0, u=t, a=\beta+(1-t)$ at $e_{5}$. A second invocation of $C_{y / 2}^{u=}$ gives $b=0, u=t, a=\beta+2(1-t)$. To reach $e x_{3}, a$ must be exactly 2 ; 
we thus need $t=\frac{\beta}{2}$. The time elapsed in one invocation of $M_{u}$ is 1 time unit; thus a total of $2+\mathrm{t}$ time units is elapsed before reaching $\ddot{*}$ (via module $C_{y / 2}^{u=}$ in $\left.\operatorname{Div}\{a, n\}\right)$. If Player 2 skips the check at $l_{2}$ and proceeds to $l_{3}$ resetting $a\left(\right.$ i.e; $y$ ), we have at $l_{3}, z=0, u=t=\frac{\beta}{2}$ and $a=0$. Only $a$ ticks at $l_{3}, a$ is supposed to "catch up" with $u$ at $l_{3}$, by elapsing $t=\frac{\beta}{2}$ in $l_{3}$. Again, at $l_{4}$, only $z$ ticks. Player 2 can verify whether $a=u$ by going to $C_{u}^{a=}$. The component $C_{u}^{a=}$ is exactly same as that in Figure 3. A time of $1-t$ is elapsed in $C_{u}^{a=}$. Thus, the time taken to reach $\stackrel{\sim}{*}$ from $C_{u}^{a=}$ is $t+t+1-t=1+t$. Thus, the exit node $e x_{2}$ of $\operatorname{Div}\{a, n\}$ is reached in time $2 t=2 \frac{\beta}{2}=\beta$. As was the case in Lemma 1 , the time taken to reach the exit node of Inc $c$, starting with $y=\beta, x=\alpha, z=0$ is $\beta+2 \frac{\alpha}{12}<2 \beta$. Also, the time taken by $\operatorname{Div}\{a, n\}$ on entering with $a=\zeta$ is $2 \frac{\zeta}{n}$.

To summarize, the time taken to reach the exit node of the Inc c component is $<2 \beta$, on entering with $y=\beta$. Also, the component $\operatorname{Div}\{a, n\}$ divides the value in clock $a$ by $n$. If $a=\zeta$ on entering $\operatorname{Div}\{a, n\}$, then upon exit, its value is $a=\frac{\zeta}{n}$. The time taken to reach the exit $e x_{2}$ is $2 *\left(\frac{\zeta}{n}\right)$. The time taken to reach the node $\ddot{*}$ in $\operatorname{Div}\{a, n\}$ is $<n+1$ (due to $n$ calls to $M_{u}$ component).

The component for zero check instruction, as in Lemma 1, divides $x$ by 6 and $y$ by 2; similarly, the component for decrement $c$ instruction divides $y$ by 2 and $x$ by 3 . The time to reach the exit node of any component corresponding to an instruction is $<2 \beta$, on enetering the component with $y=\beta$. The total time taken for the simulation of the two counter machine here also, is $<4$. Details can be found in [6].

The following lemma is an easy corollary of Lemma 1

Lemma 3 The time bounded reachability game problem is undecidable for unrestricted recursive stopwatch automata with at least 3 stopwatches.

\section{Conclusion}

The main result of this paper is that time-bounded reachability game problem for recursive timed automata is undecidable for automata with three or more clocks. We also showed that for recursive stopwatch automata the reachability problem turns undecidable even for glitch-free variant with 4 stopwatches, and the corresponding time-bounded problem is undecidable for automata with 3 stopwatches. The decidability of time-bounded reachability game for recursive timed automata with 2 clocks is an open problem.

\section{References}

[1] Parosh Aziz Abdulla, Mohamed Faouzi Atig \& Jari Stenman (2012): Dense-Timed Pushdown Automata. In: LICS, pp. 35-44. Available at http://dx.doi.org/10.1109/LICS.2012.15.

[2] Rajeev Alur, Michael Benedikt, Kousha Etessami, Patrice Godefroid, Thomas W. Reps \& Mihalis Yannakakis (2005): Analysis of recursive state machines. ACM Trans. Program. Lang. Syst. 27(4), pp. 786-818. Available at http://doi.acm.org/10.1145/1075382.1075387.

[3] Rajeev Alur \& David L. Dill (1990): Automata For Modeling Real-Time Systems. In: ICALP, pp. 322-335. Available at http://dx.doi.org/10.1007/BFb0032042.

[4] Rajeev Alur \& David L. Dill (1994): A Theory of Timed Automata. 126, pp. 183-235. Available at http: //dx.doi.org/10.1016/0304-3975(94)90010-8.

[5] Kousha Etessami (2004): Analysis of Recursive Game Graphs Using Data Flow Equations. In: VMCAI, pp. 282-296. Available at http://dx.doi.org/10.1007/978-3-540-24622-0_23. 
[6] Shankara Narayanan Krishna, Lakshmi Manasa \& Ashutosh Trivedi (2014): On The Reachability Problem for Recursive Hybrid Automata with One and Two Players. In: Manuscript, abs/1406.7289. Available at http://arxiv.org/abs/1406.7289

[7] Marvin L. Minsky (1967): Computation: finite and infinite machines. Prentice-Hall, Inc.

[8] Joël Ouaknine \& James Worrell (2010): Towards a Theory of Time-Bounded Verification. In: ICALP (2), pp. 22-37. Available at http://dx.doi.org/10.1007/978-3-642-14162-1_3.

[9] Ashutosh Trivedi \& Dominik Wojtczak (2010): Recursive Timed Automata. In: ATVA, pp. 306-324. Available at http://dx.doi.org/10.1007/978-3-642-15643-4_23.

[10] Igor Walukiewicz (1996): Pushdown Processes: Games and Model Checking. In: CAV, pp. 62-74. Available at http://dx.doi.org/10.1007/3-540-61474-5_58. 